\title{
Core Genotyping Facility
}

National Cancer Institute

\section{Source}

National Cancer Institute. Core Genotyping Facility. NCI Thesaurus. Code C48671.

A facility to perform high-throughput genotyping and sequencing to support genetic analysis for a broad range of projects. 